\title{
What is the Normal Value of Tpe Interval and Corrected Tpe Interval?
}

Hasan Koca ${ }^{1},[\mathrm{MD}]$

ORCID: 0000-0002-6232-4567

Mevlut Koc ${ }^{1},[\mathrm{MD}]$

ORCID: 0000-0002-3000-4200

\section{re) ABSTRACT Cen}

Objective: Increased T wave peak and end distance (Tpe interval) shows abnormal spread in ventricular repolarization, it is associated with increased risk of ventricular arrhythmia and poor prognosis. However, Tpe interval, corrected Tpe (Tpe-c) upper limit value, and normal reference interval have not been reported in healthy population. In our study, it was aimed to determine the normal reference range of Tpe and Tpe-c interval in healthy population.

Materials and Methods: 414 healthcare professionals who had no complaints were included in the study. Tpe and Tpe-c measurements of all patients were performed on 12-lead electrocardiography taken at rest. Average and standard deviation, median, minimum, maximum, $95 \%$ confidence intervals $(95 \% \mathrm{Cl})$ values of these parameters were obtained.

Results: According to the data obtained in our study; Tpe mean and standard deviation, median, minimum, and maximum values were found to be $70.3 \pm 12.3 \mathrm{~ms}, 70 \mathrm{~ms}, 50 \mathrm{~ms}$ and $100 \mathrm{~ms}$, respectively. The $95 \% \mathrm{Cl}$ value of the Tpe interval was detected as $50-90 \mathrm{~ms}$. Tpe-c mean and standard deviation, median, minimum, and maximum values were found to be $76.1 \pm 14.6 \mathrm{~ms}, 75 \mathrm{~ms}, 48 \mathrm{~ms}$ and $128 \mathrm{~ms}$, respectively. The $95 \% \mathrm{Cl}$ of the Tpe-c interval was detected as $53-100$ $\mathrm{ms}$. It was determined that Tpe and Tpe-c intervals were not affected by demographic and clinical parameters.

Conclusion: As a result of our study, it was determined that the upper limit of Tpe and Tpe-c interval values in adult, not-elderly and healthy normal population were $90 \mathrm{~ms}$ and $100 \mathrm{~ms}$, respectively. It was thought that this information could be used for prolonged Tpe interval, which shows an increased risk of arrhythmia in daily practice. However, it was concluded that studies should be done in other communities and races on this subject as well.

Keywords: Tpe interval, normal limit values

Corresponding Author: Hasan Koca

Specialist of Cardiology Department of Cardiology, University of Health Sciences - Adana Health Practice and Research Center, Adana, Turkey.

E-mail: hasankoca90@hotmail.com

Received: 3 August 2020, Accepted: 16 November 2020,

Published online: 31 December 2020

\section{INRODUCTION}

In electrocardiography (ECG), P - QRS - T wave occurs as the result of atrial and ventricular depolarization and repolarization, respectively. $\mathrm{P}$ wave duration, PR distance, duration of QRS waves and QT duration limit values are known and their prolongation is used in many diagnoses.

Ventricular myocardium depolarization occurs from the endocardial region to the epicardial region. Ventricular repolarization occurs following depolarization. There is dispersion between the endocardial and epicardial region during ventricular repolarization. The interval between the T wave peak and the end distance is called the Tpe interval, and this is associated with transmural ventricular repolarization [1,2]. The Tpe interval and the ratio of this interval to the QT interval have been 
shown to be associated with the arrhythmic clinical condition in many cardiac pathological conditions, and also pose a high risk for sudden cardiac death (SCD) $[3,4,5]$. Association of the increased Tpe interval and Tpe/QT ratio with arrhythmia and SCD, dispersion at repolarization between epicardial and endocardial regions of myocardium causing slow transmission in these two anatomical regions, and this situation causing an increase especially in re-entry related arrhythmia -the most common arrhythmia reason- are thought. However, as far as we have researched, there are no studies on the normal limit values of the Tpe interval and the corrected Tpe interval (Tpe-c), despite both were studied so much and that their increase clearly known to have prognostic significance.

In our study, it was aimed to determine the normal reference range of Tpe and Tpe-c interval in healthy population.

\section{MATERIALS and METHODS}

For the study, 625 health professionals working at Adana City Training and Research Hospital between the ages of 18 and 65 who had no active complaints were scanned. After the exclusion criteria, 414 healthy individuals (198 men and 216 women with a mean age of $40.4 \pm 10.3$ ) were included in the study. In the study, those are determined as the exclusion criteria: ongoing regular medicine treatment of any kind, long QT and QTc distance and QRS > $100 \mathrm{~ms}$, known syncope or sudden cardiac arrest history, acute or chronic systemic or local infection within pediatric and geriatric age group ( $<18$ age and $>65$ age), failure to perform Tpe and QTC measurements on ECG examination, known coronary artery disease (CAD) or CAD major risk factors, valvular disease, and heart failure with reduced ejection fraction. The study was carried out in line with the Helsinki Principles; and was approved by the Local Ethics Committee (Adana City Hospital, Ethics Committee, 2020).

Anamnesis and physical examinations of all individuals were performed. Age, gender, height, weight, body mass index, heart rate, and blood pressure values were recorded from demographic and clinical data.

\section{2-lead Electrocardiographic Evaluation}

12-lead ECGs obtained by MAC 2000 ECG Machine (GE medical systems information technologies, Inc., WI, USA) with a sinus rhythm of $25 \mathrm{~mm} / \mathrm{sec}$ speed and $1 \mathrm{mv} / 10 \mathrm{~mm}$ standard calibration were from the individuals included in the study. Tpe interval was defined as the time from the peak of the T wave to the point where the T wave joins and ends with the isoelectric line (Figure 1). Measurements were made primarily from V5. If V 5 is not suitable for measurement (amplitude $<1.5 \mathrm{~mm}$ ), measurements were made from $\mathrm{V} 4$ and $\mathrm{V} 6$, and their averages were taken [6]. For the QT time, the time from where the QRS started to where the T wave inverse on the isoelectric line was calculated. QT intervals were corrected for heart rate using Bazett's formula: QTC $=\mathrm{QT} /(\mathrm{RR}) 1 / 2$. Tpe interval was corrected for heart rate and Tpe-c was calculated similar to QTc interval

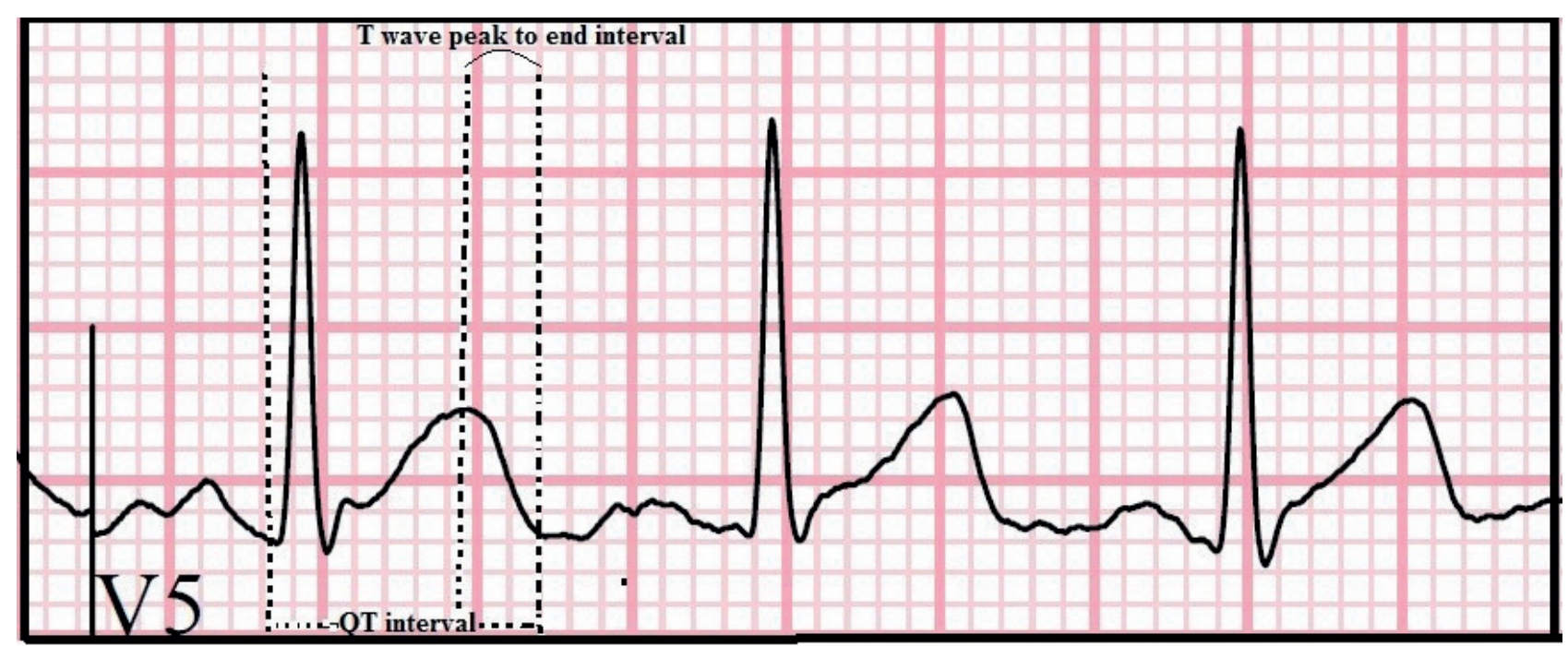

Figure 1. Normal Tpe interval measurement from V5 derivation in 12-lead electrocardiography in the healthy individual 
$[7,8]$. All the ECG examinations in sinus rhythm were evaluated by two Cardiologists with at least 5 years of experience in electrophysiology and evaluating $\geq 2000$ patients with arrhythmia annually, without knowing the identities of the individuals.

\section{Statistical Analysis}

All analyzes were performed using SPSS 22.0 (Chicago, IL, USA) statistical software package. Whether the distribution of continuous variables is normal or not was evaluated by the "KolmogorovSmirnov" test. Continuous variables were determined with mean \pm standard deviation (SD). The mean $\pm S D$, median, minimum, and maximum values of the parameters obtained with Tpe, Tpe-c, QT, QTc and their ratios were calculated. Due to the normal distribution of Tpe and QT measurements, 95\% confidence interval $(\mathrm{Cl})$ values of these parameters were given for upper and lower limit analysis. The kappa coefficient was used to examine the inter-observer and intra-observer variability of the Tpe and other ECG measurements. Continuous variables that showed normal distribution were compared using the Student $t$ test. Whether there is a relationship between countable parameters was evaluated by "Pearson's and Spearman's" correlation analysis. Statistical significance was accepted if $p$ $<0.05$.

Table 1. Interobserver and intraobserver agreements in interpreting the electrocardiographic analysis

\begin{tabular}{|l|c|c|}
\hline & $\begin{array}{c}\text { Interobserver } \\
(\%)\end{array}$ & $\begin{array}{c}\text { Intraobserver } \\
(\%)\end{array}$ \\
\hline Tpe interval (msn) & 96 & 98 \\
Tpe-c interval (msn) & 96 & 98 \\
QT interval time (ms) & 98 & 100 \\
\hline QTc interval time (ms) & 96 & 98 \\
\hline
\end{tabular}

QTc: Corrected QT, Tpe-c: Corrected Tpe

\section{RESULTS}

Tpe interval measurement was successfully performed from all individuals included in the study. Cohen kappa values that evaluate intraobserver and intra-observer variability were over $90 \%$ for all ECG measurements (Table 1 and $p<0.001$ for each-one).

The demographic and clinical parameters of the individuals included in the study are shown in Table 2. Tpe, Tpe-c, QT and QTc measurements of all individuals and mean \pm SD, median, lower, upper and $95 \% \mathrm{Cl}$ values of the rates obtained. Tpe mean and $\mathrm{SD}$, median, minimum, and maximum values were found to be $70.3 \pm 12.3 \mathrm{~ms}, 70 \mathrm{~ms}, 50 \mathrm{~ms}$ and $100 \mathrm{~ms}$, respectively. The $95 \% \mathrm{Cl}$ value of the Tpe interval was detected as 50-90 ms. Tpe-c mean and $\mathrm{SD}$, median, minimum, and maximum values were found to be $76.1 \pm 14.6 \mathrm{~ms}, 75 \mathrm{~ms}, 48 \mathrm{~ms}$ and 128 ms, respectively. The $95 \% \mathrm{Cl}$ of the Tpe-c interval was detected as 53-100 ms (Table 3). When the effect of gender on the Tpe interval is evaluated; Tpe interval in males was slightly higher than females, but this was not statistically significant $(71.4 \pm 12.6$ and $69.5 \pm 12.1$, and $p=0.099$ ). Similarly, there were no gender differences in Tpe-c, QT and QTc measurements. When the relationship between baseline demographic/clinical data and Tpe was evaluated, no parameters closely related to Tpe interval were determined.

Table 2. The clinic and demographic findings of study population

\begin{tabular}{|l|c|}
\hline Variable & \\
\hline Age (year) & $40.4 \pm 10.3$ \\
Sex (male/female) & $207 / 207$ \\
Systolic blood pressure $(\mathrm{mmHg})$ & $113 \pm 12$ \\
Diastolic blood pressure $(\mathrm{mmHg})$ & $69 \pm 9.2$ \\
Heart rate $(\mathrm{bpm})$ & $70.5 \pm 10$ \\
Body mass index $\left(\mathrm{kg} / \mathrm{m}^{2}\right)$ & $24.2 \pm 2.3$ \\
\hline
\end{tabular}

Table 3. Electrocardiographic ventricular depolarization and repolarization findings of study population

\begin{tabular}{|l|c|c|c|c|}
\hline & Mean \pm SD & Median & Lower - Upper & $95 \% \mathrm{Cl}$ \\
\hline Tpe interval (msn) & $70.3 \pm 12.3$ & 70 & $50-100$ & $50-90$ \\
\hline Tpe-c interval (msn) & $76.1 \pm 14.6$ & 75 & $48-126$ & $53-100$ \\
\hline QT interval time (ms) & $368 \pm 14$ & 365 & $340-440$ & $350-393$ \\
\hline QTc interval time (ms) & $397 \pm 24.5$ & 394 & $316-485$ & $360-440$ \\
\hline Tpe/QT ratio & $0.192 \pm 0.35$ & 0.195 & $0.130-0.290$ & $0.139-0.252$ \\
\hline Tpe/QTc ratio & $0.178 \pm 0.33$ & 0.178 & $0.110-0.280$ & $0.128-0.237$ \\
\hline Tpe-c/QT ratio & $0.208 \pm 0.43$ & 0.205 & $0.120-0.370$ & $0.146-0.281$ \\
\hline
\end{tabular}

The values were shown as mean \pm standard deviation, Cl: Confidence interval, QTc: Corrected QT, Tpe-c: Corrected Tpe SD: Standard deviation 


\section{DISCUSSION}

The main finding of our study is to determine that the normal upper reference value of Tpe and Tpe-c interval in the healthy adult population is 90 $\mathrm{ms}$ and $100 \mathrm{~ms}$, respectively. It was thought that measurements above the same reference values in both genders could be used for prolonged ventricular repolarization symptom or increased risk of arrhythmia, since the difference in both genders had no effect on the Tpe.

The QT distance covers all the ventricular depolarization and repolarization, also called the electrical systole and consists of the QRS complex, J point, ST segment and $\mathrm{T}$ wave. Ventricular myocardium depolarization occurs from the endocardial region to the epicardial region. Ventricular repolarization occurs following depolarization. The QRS complex represents ventricular depolarization, which begins in the inter-ventricular septum, proceeding from the endocardium to the epicardium and from left to right. The normal QRS complex has duration of 60-100 ms. The J point corresponds to the end of ventricular depolarization. It is located between the end of QRS complex and the beginning of the ST segment, generally on the isoelectric line. The ST segment is measured from the J point to the onset of the T wave, and it usually remains close to the isoelectric line. The phase of ventricular repolarization includes the ST segment and the $T$ wave. The latter has asymmetric ascending and descending limbs and tends to be positive in the extremity leads (except aVR) and in the precordial leads (except $\mathrm{V} 1$ ). In rare cases, the T wave is followed by a $U$ wave, which is usually positive. There is dispersion between the endocardial and epicardial region during ventricular repolarization. The interval between the $T$ wave peak and the end distance is called the Tpe interval, and this is associated with dispersion of ventricular repolarization [9]. Prolongation of the Tpe or dispersion of ventricular repolarization is associated with ventricular tachycardia and ventricular fibrillation, which are severe ventricular arrhythmia [10].

Although the QRS and QT times are clearly known from the ventricular depolarization and repolarization parameters, the normal values of the Tpe and Tpe-c interval times are not clearly known.
Only, in a recent study on healthy volunteers by Hnatkova et al. [7] did Tpe interval measurement in 12-Lead Holter recordings. As a result of this study, it was reported that the Tpe interval was significantly longer in men than in women, but it was not affected by ethnicity. In the same study, the Tpe interval has been shown to be unaffected by heart rate and age [7]. The heart rate dependency of Tpe intervals is very shallow and different subjects show both negative and positive correlations of Tpe with the different rates [7]. Although corrected Tpe according to heart rate differs numerically, it has no clinical significance, so routine correction is not recommended. However, in this study, no limit values were specified and mean \pm SD were given by all genres and ethnicity. While Caucasian female race was $87.7 \pm 6.4$, it was $94.3 \pm 5.7$ in males. Our study differs on some features according to the above-mentioned study. First, unlike the previous study, the Tpe and Tpe-c intervals were gender independent. The mean \pm SD value we obtained was approximately 10-15 ms lower than the previous study. This may be due to our assessment of 12-lead ECG withdrawal at rest. Also, Tpe interval measurement from Holter records may not be optimal.

In the published studies, different methods were used for the measurement of Tpe. The "tangent" method usually performed manually, defines the T-peak as the maximum absolute T-wave deflection from the isoelectric line, and the T-end as the intersection of the isoelectric line and the tangent to the downslope of the T-wave. Various previously published studies have used the tangent method in V2, V5, DII, and the average of measurements in V1V6 and the average of measurements in V4-V6 [3, $6,8]$. In another one, the automated GE Healthcare measurement algorithm '12SL/QT Guard Plus's (12SL, described below) also has been used in previously published studies $[8,9,10]$. Although the optimal measurement is automatic 12 lead, this may not always be achievable. In our study, because of the lack of automatic measurement, the "tangent" method and V5 were used in our study. If V5 is not suitable for measurement, measurements were made from V4 and V6, and their averages were taken. 
The Tpe interval and the ratio of this interval to the QT interval have been shown to be associated with the arrhythmic clinical condition, and also pose a high risk for sudden cardiac death $[3-5,11]$. Association of the increased Tp-e interval and Tp-e/ QT ratio with arrhythmia and SCD, dispersion at repolarization between epicardial and endocardial regions of myocardium causing slow transmission in these two anatomical regions, and this situation causing an increase especially in re-entry related arrhythmia -the most common arrhythmia reasonare thought.

In the literature, it has been reported that Tpe interval increases in coronary artery disease $[5,12$, 13], Brugada syndrome [14-16], heart failure [8, 9, 17], Short QT [18], catecholaminergic polymorphic ventricular tachycardia [19], hypertrophic cardiomyopathy [20], and brain death from noncardiac diseases [21], gastroesophageal reflux [22] anorexia nervosa [23], hypothyroidism [24], obesity [25] and primary aldosteronism [26] and this has a diagnostic and prognostic significance. In these studies, different mean \pm SD values were obtained in healthy controls taken alongside the patient groups. The most important reason for this may be the factors such as the derivation being measured, the measurement being done by manual or automatic methods, etc. It is observed that the lowest of the mean values obtained from 9 studies was $68.6 \pm 8.1 \mathrm{~ms}$ and the highest was $95.6 \pm 12.4$ ms $[25,27]$. At the Health 2000 Study (1,870 individuals) and Framingham Heart Study $(3,745$ individuals), which are two large studies involving huge number of participants with Tpe interval measurements, a Tpe interval of $73.6 \pm$ 8.4 and $95.6 \pm 12.4$ reported, respectively [27]. However, not all participants consisted of healthy controls in both studies [28]. In 2 studies containing a healthy control group conducted in our clinic, the Tpe interval value was shown to be around $70 \mathrm{~ms}$ $[21,26]$.

According to the data obtained in our study; Tpe mean and SD, median, minimum, and maximum values were found to be $70.3 \pm 12.3 \mathrm{~ms}, 70 \mathrm{~ms}, 50$ $\mathrm{ms}$ and $100 \mathrm{~ms}$, respectively. The $95 \% \mathrm{Cl}$ value of the Tpe interval was detected as 50-90 ms. Tpe-c mean and SD, median, minimum, and maximum values were found to be $76.1 \pm 14.6 \mathrm{~ms}, 75 \mathrm{~ms}$, $48 \mathrm{~ms}$ and $128 \mathrm{~ms}$, respectively. The $95 \% \mathrm{Cl}$ of the Tpe interval was detected as $53-100 \mathrm{~ms}$. As far as we have researched, there is no data on normal reference range of Tpe and Tpe-c interval in healthy population. Therefore, we could not compare our values with any study data.

\section{Limitations}

There are some important limitations in our study. Firstly, while the healthy control group taken was relatively adequate, the performed study had a single center and a single ethnicity. Regarding this kind of limit value studies, it would be better if it were done with more communities and races. Another significant limitation is the Tpe measurement method in our study. As we mentioned earlier, more accurate data could be obtained if automatic measurement was made.

\section{CONCLUSION}

The relationship of long QT and Tpe intervals with arrhythmia is clearly shown. Especially, although the QTc normal reference range is clearly known, we still do not have clear information or evidence of the normal Tpe interval. As a result of our study, it was determined that the normal Tpe and Tpe-c interval values in adult, not-elderly and healthy normal population. It was thought that this information could be used for prolonged Tpe interval, which shows an increased risk of arrhythmia in daily practice. Our current findings consist of data obtained from a specific community of a specific region of our country. For this reason, it is necessary to conduct studies with different regions, different races, and more individuals, and compare these with our data.

\section{CONFLICT OF INTEREST STATEMENT}

There is no conflict of interest. 
[1] Kongstad O, Xia Y, Liang Y, et al. Epicardial and endocardial dispersion of ventricular repolarization. A study of monophasic action potential mapping in healthy pigs. Scandinavian Cardiovascular Journal. 2005; 39: 342-7.

[2] Xia Y, Liang $Y$, Kongstad O, et al. Tpeak-Tend interval as an index of global dispersion of ventricular repolarization: evaluations using monophasic action potential mapping of the epi- and endocardium in swine. J Interv Card Electrophysiol. 2005; 14: 79-87.

[3] Porthan K, Viitasalo M, Toivonen L, et al. Predictive Value of Electrocardiographic T-Wave Morphology Parameters and T-Wave Peak to T-Wave End Interval for Sudden Cardiac Death in the General PopulationClinical Perspective. Circulation: Arrhythmia and Electrophysiology. 2013; 6: 690-6.

[4] Erikssen G, Liestol K, Gullestad L, et al. The terminal part of the QT interval (T peak to T end): a predictor of mortality after acute myocardial infarction. Ann Noninvasive Electrocardiol. 2012; 17: 85-94.

[5] Smetana P, Schmidt A, Zabel $M$, et al. Assessment of repolarization heterogeneity for prediction of mortality in cardiovascular disease: peak to the end of the T wave interval and nondipolar repolarization components. J Electrocardiol. 2011: 44: 301-8.

[6] Chua KC, Rusinaru C, Reinier K, et al. Tpeak-to-Tend interval corrected for heart rate: A more precise measure of increased sudden death risk? Heart rhythm. 2016; 13: 2181-5.

[7] Hnatkova K, Toman O, Šišáková $M$, et al. Sex and race differences in J-Tend, J-Tpeak, and Tpeak-Tend intervals. Sci Rep. 2019; 9(1): 19880.

[8] Rosenthal TM, Masvidal D, Abi Samra FM, et al. Optimal method of measuring the T-peak to T-end interval for risk stratification in primary prevention. Europace. 2018; 20(4): 698-705.

[9] Morin DP, Saad MN, Shams OF, et al. Relationships between the T-peak to T-end interval, ventricular tachyarrhythmia, and death in left ventricular systolic dysfunction. Europace 2012; 14: 1172-9.

[10] Haarmark C, Hansen PR, Vedel-Larsen E, et al. The prognostic value of the Tpeak-Tend interval in patients undergoing primary percutaneous coronary intervention for ST-segment elevation myocardial infarction. J Electrocardiol. 2009; 42: 555-60.

[11] Gupta P, Patel C, Patel H, et al. T(p-e)/QT ratio as an index of arrhythmogenesis. J Electrocardiol. 2008; 41: 567-74

[12] Hetland M, Haugaa KH, Sarvari SI, et al. A novel ECG-index for prediction of ventricular arrhythmias in patients after myocardial infarction. Ann Noninvasive Electrocardiol. 2014; 19: 330-7.

[13] Çoner A, Akıncı S, Akküçük MH, et al. Admission Tpe interval predicts reperfusion success in STEMI patients treated with fibrinolytic agents. Turk Kardiyol Dern Ars. 2020; 48(1): 49-57.

[14] Romero D, Behar N, Mabo P, et al. Differences in Brugada Syndrome Patients Through Ventricular Repolarization
Analysis During Sleep. Conf Proc IEEE Eng Med Biol Soc. 2019; 2019: 5638-41.

[15] Letsas KP, Weber R, Astheimer K, et al. Tpeak-Tend interval and Tpeak-Tend/QT ratio as markers of ventricular tachycardia inducibility in subjects with Brugada ECG phenotype. Europace. 2010; 12: 271-4.

[16] Castro Hevia J, Antzelevitch C, Torne's Barzaga F, et al. Tpeak-Tend and Tpeak-Tend dispersion as risk factors for ventricular tachycardia/ventricular fibrillation in patients with the Brugada syndrome. J Am Coll Cardiol. 2006; 47:1828-34.

[17] Lellouche N, De Diego C, Akopyan G, et al. Changes and predictive value of dispersion of repolarization parameters for appropriate therapy in patients with biventricular implantable cardioverter-defibrillators. Heart Rhythm. 2007; 4: 1274-83.

[18] Anttonen $O$, Väänänen $H$, Junttila J, et al. Electrocardiographic transmural dispersion of repolarization in patients with inherited short QT syndrome. Ann Noninvasive Electrocardiol. 2008; 13(3): 295-300.

[19] Viitasalo $M$, Oikarinen L, Väänänen $H$, et al. U-waves and T-wave peak to T-wave end intervals in patients with catecholaminergic polymorphic ventricular tachycardia, effects of beta-blockers. Heart Rhythm. 2008; 5(10): 13828.

[20] Shimizu M, Ino H, Okeie K, et al. T-peak to T-end interval may be a better predictor of high-risk patients with hypertrophic cardiomyopathy associated with a cardiac troponin I mutation than QT dispersion. Clin Cardiol. 2002; 25(7): 335-9.

[21] Demirtas AO, Urgun OD, Koca H, et al. Tp-e Interval and Tp-e/QTc Ratio Are Significantly Increased in Patients with Brain Death. Acta Medica. 2019; 50(1): 25 - 31.

[22] Asgerov E, Şenol Ö, Güler A, et al. Distribution of nucleotide variants in the DNA sequence of ERCC1 and XRCC1 genes and the effect of phenotype in patients with gastric cancer. Turk J Gastroenterol. 2019; 30(6): 517-23.

[23] Janzen $M$, Cheung CC, Steinberg $C$, et al. Changes on the electrocardiogram in anorexia nervosa: A case control study. J Electrocardiol. 2019; 56: 64-9.

[24] Findikli HA, Tutak AŞ, Aydin H. The relationship between the TSH values and the Tpeak - Tend interval duration in hypothyroid patients receiving Levothyroxine treatment. Rom J Intern Med. 2019; 57(2): 175-80.

[25] Inanir M, Sincer I, Erdal E, et al. Evaluation of electrocardiographic ventricular repolarization parameters in extreme obesity. J Electrocardiol. 2018 6; 53: $36-9$.

[26] Demirtas D, Sumbul HE, Bulut A, et al. Tp-e interval, Tp-e/ QT and Tp-e/QTc ratio in hypertensive patients with primary aldosteronism. Clin Exp Hypertens. 2020; 42(1): 93-8.

[27] Marjamaa A, Oikarinen L, Porthan $K$, et al. A common variant near the KCNJ2 gene is associated with T-peak to T-end interval. Heart Rhythm. 2012; 9(7): 1099-103. 